\title{
Comparison of the Walz Nomogram and Presence of Secondary Circulating Prostate Cells for Predicting Early Biochemical Failure after Radical Prostatectomy for Prostate Cancer in Chilean Men
}

\author{
Nigel P Murray ${ }^{1 *}$, Eduardo Reyes ${ }^{2}$, Nelson Orellana ${ }^{3}$, Cynthia Fuentealba $^{4}$, \\ Omar Jacob ${ }^{4}$
}

\begin{abstract}
Purpose: To determine the utility of secondary circulating prostate cells for predicting early biochemical failure after radical prostatectomy for prostate cancer and compare the results with the Walz nomagram. Materials and Methods: A single centre, prospective study of men with prostate cancer treated with radical prostatectomy between 2004 and 2014 was conducted, with registration of clinical-pathological details, total serum PSA pre-surgery, Gleason score, extracapsular extension, positive surgical margins, infiltration of lymph nodes, seminal vesicles and pathological stage. Secondary circulating prostate cells were obtained using differential gel centrifugation and assessed using standard immunocytochemistry with anti-PSA. Biochemical failure was defined as a PSA $>0.2 \mathrm{ng} / \mathrm{ml}$, predictive values werecalculated using the Walz nomagram and CPC detection. Results: A total of 326 men participated, with a median follow up of 5 years; 64 had biochemical failure within two years. Extracapsular extension, positive surgical margins, pathological stage, Gleason score $\geq 8$, infiltration of seminal vesicles and lymph nodes were all associated with higher risk of biochemical failure. The discriminative value for the nomogram and circulating prostate cells was high $(\mathrm{AUC}>\mathbf{0 . 8 0})$, predictive values were higher for circulating prostate cell detection, with a negative predictive value of $99 \%$, sensitivity of $96 \%$ and specificity of $75 \%$. Conclusions: The nomagram had good predictive power to identify men with a high risk of biochemical failure within two years. The presence of circulating prostate cells had the same predictive power, with a higher sensitivity and negative predictive value. The presence of secondary circulating prostate cells identifies a group of men with a high risk of early biochemical failure. Those negative for secondary CPCs have a very low risk of early biochemical failure.
\end{abstract}

Keywords: Prostate cancer - biochemical failure - circulating prostate cells - nomogram

Asian Pac J Cancer Prev, 16 (16), 7123-7127

\section{Introduction}

After radical prostatectomy for prostate cancer approximately $25-30 \%$ of patients will eventually experience a biochemical failure, defined as a total serum PSA of $>0.2 \mathrm{ng} / \mathrm{ml}$ (Hull et al., 2002; Porter et al., 2006). Two thirds of these biochemical failures will occur in the first two years and implies a biologically more aggressive disease and poorer prognosis (Dilloglugil et al, 1997). This may be due to the presence of micrometastatic disease not detected by conventional testing or from locally advanced disease (Dilloglugil et al, 1997; Pound et al, 1999; Freedland et al., 2005). Early biochemical failure is associated with a higher rate of metastatic progression and increased cancer specific mortality (Pound et al, 1999; Freedland et al., 2006). Walz et al (2009). produced a nomogram based on the total serum PSA at surgery, the presence or absence of extracapsular penetration, seminal vesicle and lymph node infiltration, positive or negative surgical margins and pathological Gleason score from the surgical piece. This externally validated nomogram, although it tends to over-estimate the risk of biochemical failure, identifies a group of men with a high risk of early biochemical failure. These individuals could be considered for adjuvant treatments, although some data have shown benefits to early therapy (MRC Working Party, 1997; Messing et al., 2006) this still is controversial.

Recent studies have shown that men positive for secondary circulating prostate cells, that is those detected after radical prostatectomy, have a seven fold increase of biochemical failure (Murray et al., 2013) and identify a group at high risk before increases in serum PSA. 
Nigel P Murray et al

We present a prospective study of Chilean men who have undergone radical prostatectomy and compare the predictions of the Walz nomogram with the presence of secondary CPCs and clinical outcomes.

\section{Materials and Methods}

Between 2008 and 2014 patients who underwent open retropubic radical prostatectomy for prostate cancer were enrolled in the stud. Pre-treatment serum total PSA was measured before digital rectal examination using the Siemens Advia CentaurXR $®$ assay. Pathological study of the surgical piece was performed by dedicated genitournary pathologists according to the Gleason system. Pathological stage was defined according to the Partin criteria, organ confined, extra capsular extension, seminal vesicle invasion and lymph node invasion (Partin et al, 2001). A positive surgical margin was defined as cancer cells in contact with the inked surface of the specimen. The registered data were entered in the on-line nomogram (www.nomogram.org) and the risk of biochemical failure in the first two years after surgery registered.

Detection of secondary circulating prostate cells: at 3 months post surgery an $8 \mathrm{~mL}$ venous blood sample was taken and collected in a tube containing EDTA (Beckinson-Vacutainer). Three months was chosen for the sampling, to give time for prostate cells disseminated during surgery would be cleared from the circulation. Samples were maintained at $4^{\circ} \mathrm{C}$ and processed within 48 hours. CPC detection was independently evaluated with the evaluators being blinded to the clinical details.

Collection of CPCs: Mononuclear cells were obtained by differential centrifugation using Histopaque 1,077 (Sigma-Aldrich), washed, and resuspended in an $100 \mu \mathrm{L}$ aliquot of autologous plasma. $25 \mu \mathrm{L}$ aliquots were used to make slides (silanized, DAKO, USA), were dried in air for 24 hours and fixed in a solution of $70 \%$ ethanol, $5 \%$ formaldehyde, and $25 \%$ phosphate buffered saline (PBS) $\mathrm{pH} 7.4$ for five mintues and finally washed three times in PBS pH 7.4.

Immunocytochemistry: secondary CPCs were detected using a monoclonal antibody directed against PSA, clone 28A4 (Novocastro Laboratory, UK), and identified using an alkaline phosphatase-anti alkaline phosphatase based system (LSAB2, DAKO, USA), with new fuchsin as the chromogen. Positive samples underwent a second process with anti-CD45 clone 2B11 + PD7/26 (DAKO, USA) and were identified with a peroxidase based system(LSAB2,DAKO, USA) with DAB (3,3 diaminobenzidine tetrahydrochloride) as the chromogen. A secondary CPC was defined according to the criteria of ISHAGE (International Society of Hemotherapy and Genetic Engineering) (Borgen et al, 1999) and the expression of CD45 as membrane bound staining. A secondary CPC was defined as a cell that expressed PSA but not CD45, a leucocyte did not express PSA but expressed CD45. A test was considered positive for secondary CPCs when at least 1 cell $/ 8 \mathrm{~mL}$ of blood was detected, the number of CPCs detected $/ 8 \mathrm{ml}$ blood simple was registered.
Statistical analysis:

Descriptive statistics were used for demographic variables, expressed as mean and standard deviation in the case of continuous variables with a normal distribution. In case of an asymmetrical distribution the median and interquartile range (IQR) values were used. Noncontiguous variables were presented as frequencies. The Shapiro-Wilk test was used to determine a normal distribution. The Student T-Test was used to compare continuous variables with a normal distribution, the Mann-Whitney test for ordinate and continuous variables with a non-normal distribution, and the Chi-squared test for the differences in frequency. Statistical significance was defined as a p value of less than 0.05 , all tests were two-sided. Area under the curve analysis was performed using the online program Vassarcalc.

The CPC test was considered positive if $\geq 1$ cell was detected per sample and negative if no cell was detected, we used a cutoff value of $20 \%$ probability of biochemical failure within two years for the Walz nomogram, based on the sensibility to detect $50 \%$ of patients who would undergo biochemical failure.

Ethical Considerations: The study was approved by the hospital ethics committee and in complete agreement with the Declaration of Helsinki. All patients provided written informed consent.

\section{Results}

A total of 326 men participated in the study, of whom 124/326 (38.0\%) suffered biochemical failure; 64/124 $(51.6 \%)$ within two years of radical prostatectomy. The clinical pathological details of men with and without biochemical failure are shown in Table 1 . The median follow up of the study population was 5 years (IQR 2-9 years).

Association of biochemical failure with clinicalpathological features:

a) Gleason score: men with higher Gleason scores were more likely to suffer biochemical failure $(\mathrm{p}<0.001)$ (Table 2).

Table 1. Demographic Features of the Study Population

\begin{tabular}{lcc}
\hline \multicolumn{1}{c}{ with biochemical failure } & without biochemical failure \\
\hline $\mathrm{N}^{\circ}$ Patients & & \\
$\quad 124$ & 202 & \\
$\begin{array}{l}\text { Mean age (SD) } \\
\quad 66.1 \pm 8.4 \text { years }\end{array}$ & $64.8 \pm 8.5$ years & $\mathrm{p}=0.38$ \\
$\begin{array}{l}\text { PSA at diagnosis ng/ml (IQR) } \\
\quad 6.52(5.13-9.79)\end{array}$ & $5.27(4.54-6.45)$ & $\mathrm{p}=0.02$ \\
\hline
\end{tabular}

Table 2. Biochemical Failure according to Gleason Score

\begin{tabular}{lccccccc}
\hline & \multicolumn{3}{c}{ Percent deviation } & & \multicolumn{3}{c}{ Standard residual } \\
\cline { 2 - 3 } \cline { 6 - 8 } & $\mathrm{BF}(+)$ & $\mathrm{BF}(-)$ & $\mathrm{BF}(+)$ & & $\mathrm{BF}(-)$ & $\mathrm{BF}(+)$ & $\mathrm{BF}(-)$ \\
\hline $\mathrm{G} \leq 6$ & 54 & 174 & $-37.50 \%$ & & $23.40 \%$ & -3.51 & 2.76 \\
$\mathrm{G} 7$ & 40 & 22 & $66.50 \%$ & & $-41.20 \%$ & 3.24 & -2.55 \\
$\mathrm{G} \geq 8$ & 30 & 4 & $130.60 \%$ & & $-80.90 \%$ & 4.71 & -3.71 \\
\hline
\end{tabular}

${ }^{*} \mathrm{G}=$ Gleason score $\mathrm{BF}=$ biochemical failure; $\mathrm{p}<0.001$ Chi squared for trends 
Walz Nomogram Secondary Circulating Prostate Cells for Predicting Early Biochemical Failure in Prostate Cancer Cases

b) pathological stage: men with higher pathological stage were more likely to suffer biochemical failure $(\mathrm{p}<0.001)$.

c) according to margen status: $34 / 124(27.4 \%)$ of men with the surgical margin positive for tumor suffered biochemical failure in comparison with 18/202 (8.9\%) of those with margins negative for tumor $(\mathrm{p}<0.0001$; RR 3.08 (95\%CI 1.82-5.20); OR 3.86 (95\%CI 2.07-7.21).

d) according to extra-capsular extension: $37 / 124$ (29.8\%) of men with extra capsular extension by tumor underwent biochemical failure in comparison with 28/202 (13.9\%) of those without extra-capsular extension $(\mathrm{p}=0.0006$; RR 2.15 (95\%CI 1.39-3.33); OR 2.64 (95\%CI 1.52-4.60).

e) seminal vesicle infiltration: $8 / 124(6.5 \%)$ of men with biochemical failure had seminal vesicle infiltration versus 0/202 without biochemical failure (Fisher exact test $\mathrm{p}=0.0004)$.

f) lymph node infiltration: $4 / 124(3.2 \%)$ of men with biochemical failure had lymph node infiltration versus 0/202 without biochemical failure) (Fisher exact test $\mathrm{p}=0.02)$.

Men with higher Gleason scores, higher pathological stage, and infiltration of the surgical margin or prostate capsule, infiltration of seminal vesicles or lymph nodes were more likely to undergo biochemical failure.

Early biochemical failure: 64/124 (51.6\%) of men underwent biochemical failure within two years of surgery, the predictive values of the Walz nomogram and secondary

Table 3. Biochemical Failure according to Pathological Stage

\begin{tabular}{lccccccc}
\hline & \multicolumn{3}{c}{ Percent deviation } & & \multicolumn{3}{c}{ Standard residual } \\
\cline { 2 - 3 } \cline { 6 - 8 } & $\mathrm{BF}(+)$ & $\mathrm{BF}(-)$ & $\mathrm{BF}(+)$ & & $\mathrm{BF}(-)$ & $\mathrm{BF}(+)$ & $\mathrm{BF}(-)$ \\
\hline pT1 & 12 & 110 & $-72.20 \%$ & & $42.30 \%$ & -4.85 & 3.71 \\
pT2 & 72 & 78 & $29.20 \%$ & & $-17.10 \%$ & 2.17 & -1.66 \\
pT3 & 40 & 24 & $69.40 \%$ & & $-40.60 \%$ & 3.37 & -2.58 \\
\hline
\end{tabular}

* $\mathrm{BF}=$ biochemical failure; $\mathrm{p}<0.001 \mathrm{Chi}$ squared for trends

Table 4. Distribution of Biochemical Failure according to Walz Nomogram and Secondary CPC Detection $(\mathrm{p}=\mathbf{0 . 5 4})$

\begin{tabular}{lccccc}
\hline $\begin{array}{l}\text { Nomogram } \\
(\% \text { risk })\end{array}$ & BF (+) & BF (-) & $\begin{array}{c}\text { CPC cells/ } \\
8 \mathrm{ml} \text { sample }\end{array}$ & BF (+) & BF (-) \\
\hline $0-5 \%$ & 6 & 192 & 0 cells & 0 & 198 \\
$6-10 \%$ & 20 & 28 & $1-2$ cells & 22 & 28 \\
$11-20 \%$ & 10 & 12 & $3-4$ cells & 18 & 16 \\
$21-50 \%$ & 12 & 24 & $5-8$ cells & 14 & 16 \\
$>50 \%$ & 16 & 6 & $>8$ cells & 10 & 2 \\
\multicolumn{7}{l}{ area under curve } & 0.805 & & & 0.834 \\
\hline
\end{tabular}

$\mathrm{CPC}$ detection were compared, men with biochemical failure occurring after two years were classified as not having biochemical failure at the two year evaluation. Table 4 shows the distribution of patients according to CPC number/8ml blood sample and \% prediction of biochemical failure.

Areas under the curve for both tests were for the nomogram 0.805 and for CPC detection 0.834 ( $\mathrm{p}=0.54$ ), there was no significant difference in the discrimative power between the two tests.

The predictive values of sensitivity, specificity, positive predictive and negative predictive values were determined for each test for different values (Table 5).

The CPC test is designed to be positive or negative; the high NPV at a cutoff of $\geq 1$ cell/sample the high negative predictive value almost excludes the risk of biochemical failure, and has a significantly higher sensitivity than a cutoff of $10 \%$ using the nomogram. There was no difference in positive predictive value, and specificity was better with the nomogram.

Theoretical treatment, over-treament and under treatment of patients based on the use of the nomogram and CPC detection.

If treatment for early biochemical failure ( $<2$ years) was recommended and based on these two specific tools to predict the individual probability of early failure after radical prostatectomy we calculated for different cut-off values the number of patients undertreated (those at risk and not treated), correctly assigned treatment (observation or active treatment) and over treatment (those not at risk but treated) (Table 6).

Using the nomogram, the use of a $6 \%$ cutoff value was inferior to that of $10 \%$ and $20 \%$ in the number of patients over treated, and superior in the under treatment of patients $(\mathrm{p}<0.001)$, there was no significant difference between a cutoff value of $10 \%$ and $20 \%(p=0.058)$. With CPC detection, although a cutoff value of 2 cells/sample was superior to that of 4 cells/sample $(\mathrm{p}<0.001)$ and mixed versus $1 \mathrm{cell} / \mathrm{sample}$, the test was designed to be positive or negative for CPCs and not based on a numerical cutoff

Table 6. Predicted Over Treatment; Adequate Treatment or Under-treatment in Individuals Based on the Individual Predictive Tests

\begin{tabular}{llccc}
\hline & Cut off value & under-T & adecuate $\mathrm{T}$ & over T \\
\hline nomogram & $\geq 6 \%$ & 6 & 250 & 70 \\
& $\geq 10 \%$ & 26 & 258 & 42 \\
& $\geq 20 \%$ & 36 & 260 & 30 \\
$\mathrm{CPC}$ & $\geq 1$ cell & 0 & 262 & 62 \\
& $\geq 2$ cells & 22 & 268 & 34 \\
& $\geq 4$ cells & 40 & 266 & 18 \\
\hline
\end{tabular}

Table 5. Predictive Values of the Walz Nomogram and Secondary CPC Detection

\begin{tabular}{|c|c|c|c|c|c|}
\hline & & sensitivity & specificity & PPV & NPV \\
\hline \multirow[t]{3}{*}{ Nomogram } & $\geq 6 \%$ & $90,6(80,1-96,1)$ & $73,3(67,4-78,5)$ & $45,3(36,5-54,3)$ & $97,0(93,2-98,8)$ \\
\hline & $\geq 10 \%$ & $59,4(46,3-71,2)$ & $84,0(78,8-88,1)$ & $47,5(36,3-58,9)$ & $89,4(84,7-92,9$ \\
\hline & $\geq 20 \%$ & $43,8(31,6-56,3)$ & $88,6(83,9-92,0)$ & $48,3(35,1-61,7)$ & $86,6(81.8-90,3)$ \\
\hline \multirow[t]{3}{*}{ СРC } & $\geq 1 \mathrm{cell} / \mathrm{sample}$ & $96,4(90,4-99,9)$ & $75,5(69,8-80,6)$ & $50,3(41,1-56,9)$ & $99,4(96,8-99,9)$ \\
\hline & $\geq 2$ cells/sample & $65,6(52,6-76,8)$ & $86,9(82,1-90,7)$ & $55,3(43,5-66,5)$ & $91,1(86,7-94,2)$ \\
\hline & $\geq 4$ cells/sample & $37,5(26,0-50,5)$ & $93,1(89,1-95,7)$ & $57,1(41,1-71,9)$ & $85,8(81,1-89,6)$ \\
\hline
\end{tabular}


Table 7. Predicted Over Treatment, Adecuate Treatment or Under Treatment in Individual Based on Combined Predictive Test

\begin{tabular}{lccc}
\hline $\begin{array}{c}\text { Nomogram }+ \text { CPC (+) } \\
\text { Cut off value }\end{array}$ & under-T & adequate-T & over-T \\
\hline$\geq 6 \%$ CPC $(+)$ & 6 & 286 & 34 \\
$\geq 10 \%$ CPC $(+)$ & 18 & 286 & 22 \\
$\geq 20 \%$ CPC $(+)$ & 38 & 270 & 18 \\
\hline
\end{tabular}

value of $\mathrm{x}$ cells/sample.

Combining the same cutoff values for the nomogram and CPC (+) gave the following results (Table 7).

The area under the curve for the combined test was 0.668 , significantly inferior to either test whether used alone $(\mathrm{p}=0.001$ versus $C P C s$, and $\mathrm{p}=0.01$ versus nomogram).

\section{Discussion}

In the study population the standard risk factors for predicting disease progression were significantly higher in the early biochemical failure group, confirming that the study population is a representative group of those who will experience progression after radical prostatectomy.

Early recurrence after radical prostatectomy is associated with a poor prognosis with early progression both in terms of metastasis and cancer specific mortality (Freedland et al., 2005; Pound et al., 2005). Therefore identifying these patients at high risk of early relapse may have clinical applications, these patients being considered for adjuvant therapies. It must be emphasized that the use of adjuvant therapy is controversial, with conflicting results reported in the literature (Mitchell et al., 2008). Clinical decisions should be based on an understanding of the various parameters which play a role in defining the risk of disease progression. The use of nomograms integrates these variables to predict patient outcome on an individual basis, rather than categorizing the patient as part of a risk group.

The nomogram designed by Walz et al, to predict early biochemical failure uses easily acquired parameters, and a predictive value of $>0.80$, the same as for the detection of secondary CPCs. Its high negative predictive value using a cutoff of $>6 \%$ suggests that men with low risk of biochemical failure do not need frequent follow up, however as it is a continuous variable the selection of an ideal cutoff percentage has not been determined, and may differ in different racial populations.

It also may not represent the dynamic process of cancer progression; the parameters of the nomogram are fixed; being determined by the pathological parameters of the surgical specimen. The phenotypic characteristics of a cancer may change as a result of treatment or mutation, therefore tumor characteristics present at surgery and seen as the clinical-pathological variables may change with disease progression or there may be differences in phenotypic expression between the primary tumor and metastasis (Goranova et al., 2011; Brodsky et al., 2014). This instability of tumor cells may be present at the time of diagnosis or as a result of clonal selection due to treatment (Zeijlemaker et al., 2014). Secondary CPCs arise from local or systemic mircometastatic foci, thus may not represent the primary tumor, changes in the expression of different biomarkers by CPCs, such as HER-2, may indicate the need to change treatments (Murray et al., 2011).

The detection of secondary CPCs is a dynamic process, changes in the number of secondary CPCs, or the disappearance or appearance of CPCs may reflect biological changes in the disease. The ability to repeat the test means that they may also be used to monitor the effects of treatment. Secondary CPC negative men had a very low risk of biochemical failure; and thus these men may need less frequent follow up, having been "cured "of their disease or are at risk of late failure. Men positive for secondary CPCs would need more frequent follow up in the first 2 years owing to the high risk of failure. the expression of different biomarkers by CPCs, such as HER-2, may indicate the need to change treatments (Murray et al., 2011).

The combined used of nomogram and secondary CPCs had a lower predictive value of identifying patients with a high risk of failure, this could suggest that the tests reflect different tumor characteristics which indicate the risk of disease progression.

However, it is important to emphasis although the nomogram and secondary CPC detection have a high predictive value of those patients with risk of early biochemical failure, neither of them predict whether the failure will be local or distant. This means that whatever treatment decision is made, the tests do not indicate whether the risk is local failure where radiotherapy may be used or distant failure whether hormonal manipulation may be more appropriate.

In conclusion, the detection of secondary CPCs in men after radical prostatectomy for prostate cancer identifies those with a high risk of early biochemical failure but does not determine whether the micrometastatic foci from where they arise is local or distant from the prostate bed. Men negative for secondary CPCs have a very low risk of biochemical failure and therefore may not need intensive follow up. Phenotypic characterization of secondary CPCs may help in selecting the most appropriate systemic therapy.

\section{Acknowledgements}

The authors wish to thank Mrs. Ana Maria Palazuelos for her patientce and help with the writing of this manuscript.

\section{References}

Borgen E, Naume B, Nesland JM, et al (1999). Standardization of the immunocytochemical detection of cancer cells in $\mathrm{BM}$ and blood. I. Establishment of objective criteria for the evaluation of immunostained cells. Cytotherapy, 1, 377-88.

Brodsky AS, Fischer A, Miller DH, et al (2014). Expression profiling of primary and metastatic ovarian tumors reveals differences indicative of aggressive disease. PLoS One, 14, 94476 
Walz Nomogram Secondary Circulating Prostate Cells for Predicting Early Biochemical Failure in Prostate Cancer Cases

Dillioglugil O, Leibman BD, Kattan MW, et al (1997). Hazard rates for progression after radical prostatectomy for clinically localized prostate cancer. Urology, 50, 93

Freedland SJ, Humphreys EB, Mangold LA, et al (2005). Risk of prostate cancer specific mortality following biochemical recurrence after radical prostatectomy. JAMA, 294, 433

Goranova TE, Ohue M, Shimoharu Y, et al (2011). Dynamics of cancer cell subpopulations in primary and metastatic colorectal tumors. Clin Exp Metastasis, 28, 427-35

Hull GW, Rabanni F, Abbas F, et al (2002). Cancer control with radical prostatectomy alone in 1,000 consecutive patients. J Urol, 167, 528

Messing EM, Manola J, Yao J, et al (2006). Immediate versus delayed androgen depreviation treatment in patients with node positive prostate cancer after radical prostatectomy and pelvic lymphadenectomy. Lancet Oncol, 7, 472

Mitchell RE, Chang SS (2008). Current controversies in the treatment of high risk prostate cancer. Curr Opin Urol, 18, 263

Murray NP, Badinez L, Dueñas R, et al (2011). Positive HER-2 expression in circulating prostate cells and micrometastasis. Resistent to androgen suppression but not DES. Ind J Urol, 27, 200-206.

Murray NP, Reyes E, Orellana N, et al (2013). Secondary circulating prostate cells predict biochemical failure after radical prostatectomy and without evidence of disease. The Scientific World J; http://dx.doi.org/10.1155/2013/762064

Partin AW, Mangold LA, Lamm DM, et al (2001). Contemporary update of prostate cancer staging nomograms (Partin tables) for the new millennium. Urol, 58, 843

Porter CR, Kodama K, Gibbons RP, et al (2006). 25 year prostate cancer control and survival outcomes, a 40 year radical prostatectomy single institution series. J Urol, 176, 569

Pound CR, Partin AW, Eisenberger MA, et al (1999). Natural history of progression after PSA elevation following radical prostatectomy. JAMA, 281, 1591

The MRC Prostate Cancer Working Party Investigators Group (1997). Immediate versus deffered treatment for advanced prostate cancer; initial results of the MRC Trial. Br J Urol, 79, 235

Walz J, Chun FK, Klein EA, et al (2009). Nomogram predicting the probability of early recurrence after radical prostatectomy for prostate cancer. $J$ Urol, 181, 601-8.

Zeijlemaker W, Gratama JW, Schuurhuis GJ (2014). Tumor heterogeneity makes AML a "moving target" for detection of residual disease. Cytometry B Clin Cytom, 86, 3-14 